\title{
Investigating the Lateral Restraining Stiffness Effect on the Bridge Deck-Wave Interaction under Stokes Waves
}

\author{
Guoji Xu, A. M. ASCE ${ }^{1}$, C.S. Cai, P.E., F. ASCE ${ }^{2}$
}

${ }^{1}$ Center for Computation \& Technology, Louisiana State Univ., Baton Rouge, 70803; Division of Computer Science and Engineering, Louisiana State Univ., Baton Rouge, LA 70803. (Corresponding author) E-mail: gxu2@1su.edu

${ }^{2}$ Edwin B. and Norma S. McNeil Distinguished Professor, Dept. of Civil and Environmental Engineering, Louisiana State Univ., Baton Rouge, LA 70803. E-mail: cscai@ lsu.edu

Abstract: This paper addresses one fundamental question on the bridge deck-wave interaction:

do the bridge deck vibrations result in smaller wave forces on deck? In other words, are there any

benefits to the coastal bridge structures vulnerable to waves once the bridge superstructure (deck)

is allowed to vibrate to some extent, similar to the lateral restraining setups adopted to bridges in seismic areas. Based on a methodology of the mass-spring-damper system using a dynamic mesh updating technique proposed in previous studies, a wave model based on the Stokes $2^{\text {nd }}$ order wave theory is applied and verified with analytical solutions and experimental measurements in the literature, assuring a valid prediction in the following parametric study. The shear stress transport (SST) $k-\omega$ model is used as the turbulence closure for the RANS equations in order to capture the turbulence effects in the bridge deck-wave interaction. In the parametric study, general characteristics of the structural vibration and the wave forces are observed and discussed. The obtained results show that increasing the structural flexibilities in the transverse/lateral direction does not necessarily benefit the bridge structure with an obvious force reduction for both the horizontal and vertical forces on the bridge superstructure. However, this will unfortunately lead to larger horizontal forces on the interface between the bridge super and substructure and larger dynamic amplification factors for the bridge decks.

Key words: Bridge deck-wave interaction; Coastal bridges; Wave forces; Computational fluid dynamics (CFD); Lateral restraining stiffness; Stokes wave. 


\section{Numerical investigation of the Lateral Restraining Stiffness Effect on the Bridge Deck- Wave Interaction under Stokes Waves}

${ }^{1}$ Center for Computation \& Technology, Louisiana State Univ., Baton Rouge, 70803; Division

5 of Computer Science and Engineering, Louisiana State Univ., Baton Rouge, LA 70803. 6 (Corresponding author) E-mail: gxu2@1su.edu

$7{ }^{2}$ Edwin B. and Norma S. McNeil Distinguished Professor, Dept. of Civil and Environmental 8 Engineering, Louisiana State Univ., Baton Rouge, LA 70803. E-mail: cscai @ lsu.edu

Abstract: This paper addresses one fundamental question on the bridge deck-wave interaction:

11 do the bridge deck vibrations result in smaller wave forces on the deck? In other words, are there

12 any benefits to the coastal bridge structures vulnerable to waves once the bridge superstructure

13 (deck) is allowed to vibrate to some extent, similar to the lateral restraining setups adopted to

14 bridges in seismic areas? Based on a methodology of the mass-spring-damper system using a

15 dynamic mesh updating technique proposed in previous studies, a wave model of the Stokes $2^{\text {nd }}$

16 order wave theory is applied and verified with analytical solutions and experimental

17 measurements in the literature, assuring a valid prediction in the following parametric study. The

18 shear stress transport (SST) $k$ - $\omega$ model is used as the turbulence closure for the RANS equations

19 in order to capture the turbulence effects in the bridge deck-wave interaction. In the parametric

20 study, general characteristics of the structural vibration and the wave forces are observed and

21 discussed. The obtained results show that increasing the structural flexibilities in the

22 transverse/lateral direction does not necessarily benefit the bridge structure with an obvious force

23 reduction for both the horizontal and vertical forces on the bridge superstructure. However, this

24 will unfortunately lead to larger horizontal forces on the interface between the bridge super and

25 substructure and larger dynamic amplification factors for the bridge decks.

26 Key words: Bridge deck-wave interaction; Coastal bridges; Wave forces; Computational fluid

27 dynamics (CFD); Lateral restraining stiffness; Stokes wave. 


\section{Introduction}

The bridge deck-wave interaction has become a focal point recently because many coastal low-lying bridges along the Gulf of Mexico in the United States have been destroyed in

31 the last decade [1-4]. The combination of the high storm surge and the high waves induced by

32 hurricanes inundated many spans and exerted huge hydrodynamic wave loads on the bridge

33 decks. However, those damaged bridges were rarely designed for such kinds of wave forces. To

34 reveal the failure mechanisms of the damaged bridges and to propose potential guidelines for

35 better designing or retrofitting coastal bridges under such extreme natural events, many

36 experimental and numerical studies have been conducted [5-12]. However, most of these studies

37 focus on the rigid setups where the bridge model is fixed in the wave flume/tank experimentally

38 and computationally. Very few studies have investigated the dynamic characteristics (flexible

39 setups) of the bridge deck-wave interaction [9, 13-15].

40 Dynamic analysis for bridge deck-wave interaction problems is an emerging topic and it

41 is of significant importance. There are two necessities on this topic: (1) the bridge deck should

42 vibrate under the wave actions due to the structure's flexibility. The structural vibration period in

43 the transverse/lateral direction is typically small for low-lying coastal bridges, say, less than $1 \mathrm{~s}$

44 [9]. However, the bridge-scour developed during the wave actions may increase the structural

45 vibration period and hence result in a larger vibration displacement under the same wave

46 conditions; and (2) similar to the mitigation ideas learned from earthquake engineering where

47 memory alloys, base isolations, and cable restraints are used to modify the interface stiffness

48 between the super and substructure in order to reduce the damage to the bridges, the bridge

49 superstructure (deck) can be allowed to vibrate to some extent such that the wave forces would

50 be reduced. There were some discussions that using flexible connections between the super and 
51 substructures may reduce the wave induced deck forces [3]. This is based on the assumption that

52 a larger lateral displacement of the bridge deck along the wave propagation would dissipate more

53 wave energy. However, the general observations made by Bradner et al. [9] did not support this

54 assumption. Thus, to reach a general consensus, a systematic dynamic analysis of the bridge

55 deck-wave interaction is essential for designing and retrofitting coastal bridges that will

56 experience hurricane events in their service period.

Fig. 1 shows a schematic diagram for the bridge deck-wave interaction under periodical

58 waves, where the superstructure movement results from the substructure flexibility and the

59 interface movement between the super and substructure. The interface portion is not plotted in

60 this figure for clarity. The bridge superstructure is deemed as a large concentrated mass at the top

61 of the cantilever type pile systems and would vibrate much laterally once sufficient wave forces

62 were exerted on them. Bradner et al. [9] developed a flexible experiment setup for the bridge

63 superstructure (a single degree of freedom (SDOF) system) using springs to represent the

64 structural stiffness, where the total lateral restraining stiffness is concerned, without

65 distinguishing the stiffness from the interface or substructure. Quite limited data was reported;

66 however, some general observations were made.

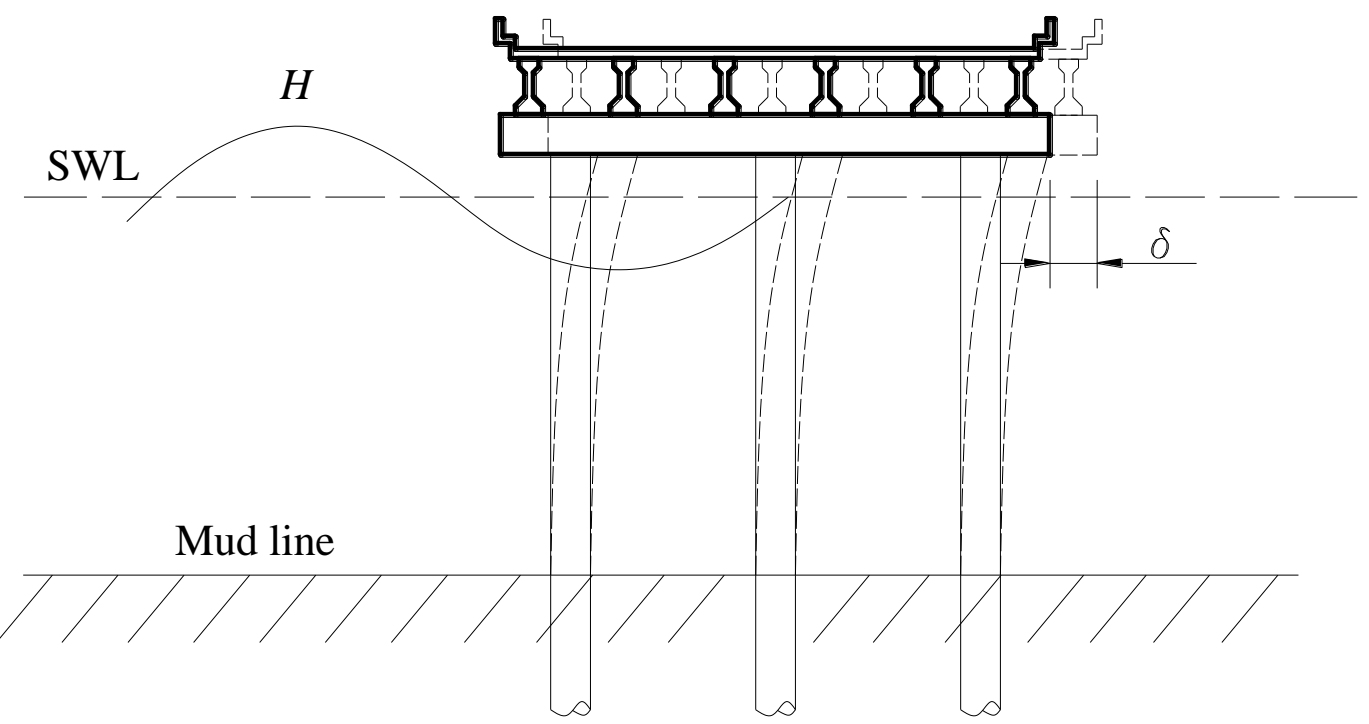


68 Fig. 1 Schematic diagram for the bridge deck-wave interaction under hurricane induced waves.

$69 H$ refers to the wave height; $\delta$ is the structural displacement for the bridge deck; SWL refers to

70 the still water level; Mud line refers to where the piles can be deemed as rigid supported. The interface is not plotted for clarity.

In the literature, many wave models (e.g., cnoidal wave, Stokes wave, solitary wave, and

74 stream function wave) have been utilized to investigate the bridge deck-wave interaction

75 problems. These wave models can be classified into two typical types: one is the solitary wave

76 model with theoretically infinite wave length and wave period; and the other is the periodical

77 wave model with finite wave length and wave period. The wave forces on deck by employing

78 different wave models should have some differences. For example, given the same wave height,

79 the wave forces of a cnoidal wave with a large wave length are in good agreement with those of

80 a solitary wave; however, there are differences between the results of these two wave models

81 when the cnoidal wave has a comparably smaller wave length [16]. The main reason is that one

82 single wave in the cnoidal wave trains with a large wave length is similar to a solitary wave in

83 general wave shape. However, it should be noted that part of the cnoidal wave is under the SWL,

84 but a solitary wave is fully above the SWL [16]. As such, the results of solitary waves may not

85 be directly used for those coastal bridges subjected to periodical waves with relatively small

86 wave lengths. The structural vibration induced by solitary waves decreases all the way to 0 ,

87 whereas the vibration induced by periodical waves during one wave period may be further

88 incited by the following incident waves. Hence, based on previous studies considering solitary

89 wave actions on flexible bridge decks [13], the present study specifically investigates the flexible

90 bridge decks under one typical periodical wave model - Stokes $2^{\text {nd }}$ order wave.

91 Bearing the fundamental question in mind, the objective of the present study is to

92 investigate the lateral restraining stiffness effects on the bridge deck-wave interaction under

93 Stokes $2^{\text {nd }}$ order waves that can represent hurricane induced waves. At first, the wave model 
94 based on the Stokes $2^{\text {nd }}$ order wave theory is applied and verified with analytical solutions and

95 experimental measurements in the literature. Using a dynamic mesh updating technique, a mass-

96 spring-damper system is realized in a commercial CFD program (Fluent, Academic Version,

97 V15.0) [17] to represent the bridge deck vibrations in the transverse/lateral direction under stokes

98 waves. Then, a parametric study is conducted and general characteristics of the structural

99 vibration and the wave forces are observed and discussed. Conclusions are finally given.

100 2. Numerical Method and Experimental Verification

$101 \quad 2.1$ Realization of the Mass-Spring-Damper System

102 Due to the complexity of this physical problem, there are limitations for adequately

103 describing the bridge superstructure system and for realizing the procedure of the bridge deck-

104 wave interaction with sufficient accuracy. In order to obtain an appropriate balance among the

105 physical realities, model sophistication, and computational cost, two dimensional (2D)

106 simulations that are widely adopted in the literature for this topic are used in the present study.

107 The bridge deck model for flexible setups can be deemed as a mass-spring-damper system (as

108 shown in Fig. 2) in order to accommodate the SDOF system. As shown in Fig. 2, the whole

109 computational domain is partitioned into three parts, two fixed zones and one remeshing zone,

110 and the mesh in the fixed zones (zones 1 and 2) remains the same as its original mesh. The mass-

111 spring-damper system (Fig. 2(b)) is incorporated in the remeshing zone (Fig. 2(a)) and their

112 combination is treated as a rigid body, which will move laterally in both directions using the

113 layering mesh method (Fig. 2(c)). For the layering mesh method, height based method is chosen

114 in the current study. The cell layer adjacent to the moving boundary, i.e., layer $j$, is split or

115 merged with the cell layer next to it, i.e., layer $i$, based on the cell height in layer $j\left(h_{j}\right)$. When

116 the bridge deck moves to the left side, the cells in layer $j$ are expanding and their height is 
117 allowed to increase if $h_{\min }>\left(1+\alpha_{s}\right) h_{\text {defined }}$, where $h_{\min }$ is the minimum cell height for layer $118 j, h_{\text {defined }}$ is the predefined cell height with a constant value, and $\alpha_{s}$ is the split factor. When the 119 bridge deck moves to the right side, the cells in layer $j$ are being compacted and they can be 120 compacted if $h_{\min }<\alpha_{c} h_{\text {defined }}$, where $\alpha_{c}$ is the collapse factor. Once the condition is met, the 121 cells in layer $j$ are split into two layers, i.e., the new layers $i$ and $j$, or merged with those in the 122 adjacent cell layer $i$ into one layer, i.e., the new layer $j$. The split factor and collapse factor used 123 are 0.4 and 0.2 , respectively.

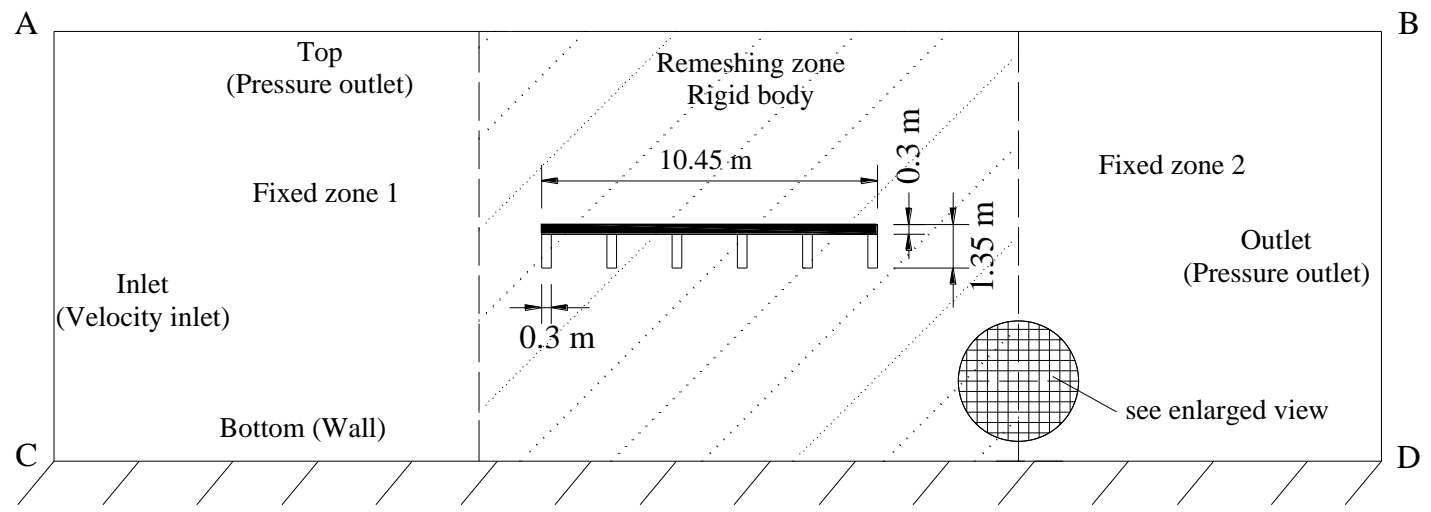

(a) Remeshing zone and Fixed zone in the computational domain

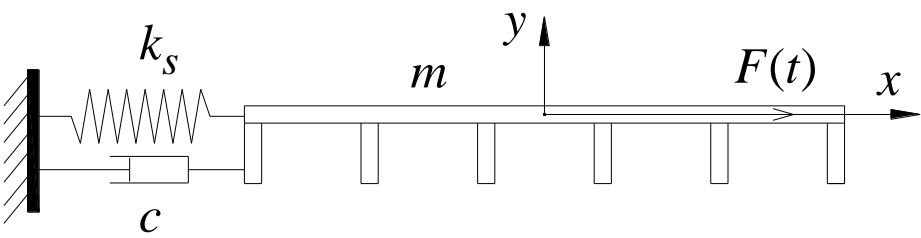

(b) The mass-spring-damper system 

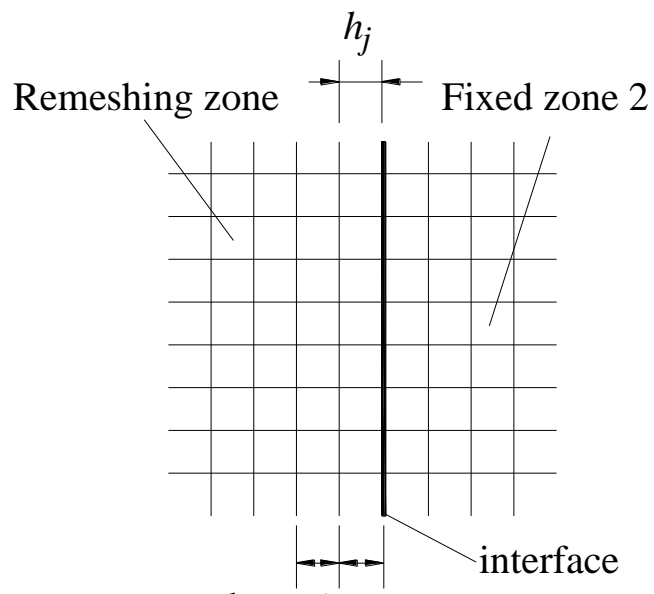

layer $i$ layer $j$

(c) Enlarged view for the layering mesh method

130 Fig. 2 Schematic diagram of the computational domain for the mass-spring-damper system. In 131 this system, $m$ is the unit length weight of the bridge deck, $k_{s}$ is the lateral restraining stiffness, and $c$ is the damping coefficient.

133 The boundary conditions in the computational domain are as follows: the line $\mathrm{AB}$ is

134 pressure outlet, keeping the pressure in the air phase the same as the operating pressure (101, 325

$135 \mathrm{~Pa})$; the line $\mathrm{AC}$ is velocity inlet; the bottom line $\mathrm{CD}$ is modeled with a no slip stationary wall

136 condition; and the line BD is set as pressure outlet. The water phase in the computational domain

137 for the prescribed wave conditions will be patched through the Fluent at the initialization stage.

138 The sketch of the geometry of a typical coastal bridge deck model is also shown in Fig. 2. This

139 prototype bridge, which will be used in the parametric study, consisting of a slab and six

140 AASHTO type III girders, is designed to carry two traffic lanes on the deck and can be

141 commonly found connecting coastal communities [7, 18]. The width of the superstructure is

$14210.45 \mathrm{~m}$, the girder height is $1.05 \mathrm{~m}$, and the slab depth is $0.3 \mathrm{~m}$. All six of the girders, each with

143 a width of $0.3 \mathrm{~m}$, are simplified as rectangles and evenly distributed.

144 The vibration of the bridge deck in the $x$ /lateral direction can be described as the 145 following equations: 
147 where $x$ is the deck displacement in the $x$ direction, $\xi$ is the damping ratio $\left(c=2 \xi \omega_{0} m\right), \omega_{0}$ is

148 the natural frequency of the bridge deck $\left(\omega_{0}=2 \pi / T_{s}\right), F(t)$ is the horizontal force on the bridge 149 deck, and $T_{S}$ is the structural vibration period. Therefore, based on the known mass, vibration 150 period, and the damping ratio of the bridge structure and the instantaneous horizontal force on 151 the deck, the corresponding structural vibrations can be obtained. Regarding the lateral vibration 152 of the bridge deck, the utilized SDOF system is more suitable for simply supported coastal low153 lying bridges than continuously supported and long span ones which are deemed as having 154 involvement of multiple degree of freedoms. As a matter of fact, it seems very difficult, if not 155 impossible, currently to simulate the whole bridge superstructure in the computational domain in 156 order to catch the features of multiple degree of freedoms due to the limitation of the 157 computational power. However, since the lateral fundamental frequency (usually the 1st lateral 158 mode) is the most important one for the lateral vibration, it can be decoupled from the other 159 modes. Therefore, the SDOF system serves well for the demonstration purpose of the present 160 study. The details regarding the realization and verification of the mass-spring-damper system 161 can be found in Xu and Cai [13] and Xu et al. [14].

\section{$162 \quad 2.2$ Wave Generation and Verification with Analytical Results}

163 The bridge deck-wave interaction is of high turbulent features, where transported 164 quantities, e.g., mass and momentum, fluctuate in time and space. The wave impact on the bridge 165 superstructure is unsteady and stochastic, and it is actually unpredictable in detail. In this sense, 166 turbulent models should be essentially preferred for this bridge deck-wave interaction topic. 167 However, in order to simplify the problem, the laminar model can be used as a first 168 approximation. The results in $\mathrm{Xu}$ et al. [14] show that there are no big differences between the 
170 using the laminar model can make a good approximation in some cases. However, in cases, for

171 example, with high Reynolds number and for the wave breaking (say, wave run-up), the laminar

172 model would produce unreliable results.

173 To capture the turbulent features for the bridge deck-wave interaction, the shear stress

174 transport (SST) $k-\omega$ model is used as the turbulence closure for the RANS equations. The

175 reasons for choosing this turbulent model over the $k-\varepsilon$ model and the Large Eddy Simulation

176 (LES) model are: (1) While the $k-\varepsilon$ model is one of the most common turbulence model, it is

177 more suitable for the flow with high Reynold numbers. However, the flow near the walls of the

178 bridge model tends to be characterized as the low Reynold number flow, which may lead to

179 unrealistic results for the $k-\varepsilon$ model. Though many wall functions can be therefore employed for

180 the $k-\varepsilon$ model to make up this shortcoming, the SST $k$ - $\omega$ model has its advantages over the $k-\varepsilon$

181 model such that the flow domain with a high Reynold number and the near wall domain with a

182 relatively low Reynold number can be more appropriately resolved; and (2) The LES model

183 requires more strictly for the grid mesh in the computational domain, especially for the near wall

184 mesh, i.e., $y+\leq 1$, where $y+$ is used to calculate the height of the first grid cell along the walls.

185 As such, LES model is much more computationally expensive, though it can yield more

186 turbulent features. The equations for the SST $k-\omega$ model are given as follows:

$187 \quad \frac{\partial}{\partial \mathrm{t}}(\rho k)+\frac{\partial}{\partial x_{i}}\left(\rho k u_{i}\right)=\frac{\partial}{\partial x_{j}}\left(\Gamma_{\mathrm{k}} \frac{\partial k}{\partial x_{j}}\right)+\widetilde{\mathrm{G}_{k}}-\mathrm{Y}_{k}+\mathrm{S}_{k}$

$188 \frac{\partial}{\partial \mathrm{t}}(\rho \omega)+\frac{\partial}{\partial x_{j}}\left(\rho \omega u_{j}\right)=\frac{\partial}{\partial x_{j}}\left(\Gamma_{\omega} \frac{\partial \omega}{\partial x_{j}}\right)+\mathrm{G}_{\omega}-\mathrm{Y}_{\omega}+\mathrm{D}_{\omega}+\mathrm{S}_{\omega}$

189 where $k$ is turbulent kinetic energy which determines the energy in the turbulence, $\omega$ is the 190 specific dissipation which determines the scale of the turbulence; $\Gamma_{k}$ and $\Gamma_{\omega}$ are the effective 
191 diffusivity of $k$ and $\omega$, respectively; $\widetilde{\mathrm{G}_{k}}$ represents the generation of turbulence kinetic energy

192 due to the mean velocity gradients, calculated from $\mathrm{G}_{k} ; \mathrm{G}_{\omega}$ is the generation of $\omega ; \mathrm{Y}_{k}$ and $\mathrm{Y}_{\omega}$ are

193 the dissipation of $k$ and $\omega$, respectively; $\mathrm{D}_{\omega}$ is the cross-diffusion term; and $\mathrm{S}_{k}$ and $\mathrm{S}_{\omega}$ are user-

194 defined source terms.

195 In the present study, the Stokes $2^{\text {nd }}$ order wave theory is employed to represent one 196 typical wave type induced by hurricanes. The water particle velocities $u$ and $v$ and the free

197 surface profile $\eta$ of the Stokes $2^{\text {nd }}$ order wave theory are expressed as follows [19]:

$198 u=\frac{H}{2} \frac{g K}{w} \frac{\cosh K(h+z)}{\cosh K h} \cos (K x-w t)+\frac{3 H^{2} w K}{16} \frac{\cosh 2 K(h+z)}{\sinh ^{4}(K h)} \cos 2(K x-w t)$

199

$v=\frac{H}{2} \frac{g K}{w} \frac{\sinh K(h+z)}{\cosh K h} \sin (K x-w t)+\frac{3 H^{2} w K}{16} \frac{\sinh 2 K(h+z)}{\sinh ^{4}(K h)} \sin 2(K x-w t)$

$200 \quad \eta=\frac{H}{2} \cos (K x-w t)+\frac{H^{2} K}{16} \frac{\cosh (K h)}{\sinh ^{3}(K h)}(2+\cosh 2 K h) \cos 2(K x-w t)$

201 where $K$ is the wave number, $w$ is the wave frequency, $h$ is the still water depth, $g$ is the 202 gravitational acceleration, $z$ is the distance from the still water level and is negative if it has the 203 same direction as the gravitational acceleration, $t$ is the simulation time, and $x$ is the distance 204 from the inlet boundary.

205 In the simulations, water is assumed to be an incompressible, viscous fluid, and the 206 Volume of Fluid (VOF) method is utilized to track the dynamic free surface [17, 20]. The VOF 207 model is a surface-tracking method applied for two or more immiscible fluids where the position 208 of the interface between the fluids is the focal point. In this VOF model, a single set of 209 momentum equations are solved for all the shared fluids and the volume fraction for each of the 210 shared fluids is tracked in the whole computational domain. For the two phase problems, $\alpha_{q}=1$ 211 refers to that the mesh cell is fully occupied with the $q$ th phase, which is the primary phase, and $212 \alpha_{q}=0$ means that the other phase fluid, the secondary phase, occupy the very mesh cell. 
213 However, $0<\alpha_{q}<1$ indicates that the mesh cell is shared by both fluids with their respective

214 occupation ratio. In the current bridge deck-wave interaction simulations, air is set as the primary

215 phase and water is the secondary phase, and $\alpha_{q}$ is determined by the equations as follows:

$216 \frac{\partial \alpha_{q}}{\partial \mathrm{t}}+\frac{\partial\left(u \alpha_{q}\right)}{\partial \mathrm{x}}+\frac{\partial\left(\mathrm{v} \alpha_{q}\right)}{\partial \mathrm{z}}=0(q=1,2)$

$217 \quad \sum_{q=1}^{2} \alpha_{q}=1$

218 It should be noted that the pressure-based solver should be chosen when the VOF method is 219 employed and the VOF method is not available with the density-based solver.

220 For the setups of the SST $k-\omega$ model in the Fluent, the pressure-based solver (segregated)

221 is chosen for the transient flow, and the Pressure-Implicit with Splitting of Operators (PISO)

222 scheme is utilized for the pressure-velocity coupling method. Further details of the numerical 223 setups can be found in $\mathrm{Xu}$ and Cai [13].

224 For the wave generation, the equations of the velocity components, $u$ and $v$, and the free 225 surface profile $\eta$ are compiled into the Fluent at the inlet boundary (see Fig. 2 (a)) 226 simultaneously using the user defined function (UDF) macros of DEFINE_PROFILE. A 227 numerical flume with the size of $55 \mathrm{~m}$ in length and $3.2 \mathrm{~m}$ in height is chosen for the verification 228 of the wave profiles with analytical results. Based on the mesh sensitivity study by $\mathrm{Xu}$ and Cai 229 [13] and many previous studies (e.g., [7, 8, 18, 21]), several summarized guidelines need to be 230 followed for successful simulations of the bridge deck-wave interaction: (1) To well represent 231 the surface waves, sufficient grids need to be considered per the wave length and wave height. 232 Practically speaking, 100 nodes in one wave length and 20 nodes in the wave height can well 233 predict the wave shape. However, high nonlinear waves may need more dense meshes; (2) The

234 Courant-Friedrichs-Lewy condition needs to be strictly satisfied; (3) The skewness of the cell, $\frac{d y}{d x}$ 
235 or $\frac{d x}{d y}$, needs to be smaller than 10 [17]. However, smaller values, say, 1 2, would produce more 236 accurate results; and (4) To accurately capture the near-wall features, very fine meshes should be 237 adopted around the walls of the bridge deck model in order to satisfy the requirement of the log238 law for the "law-of-the-wall". Further elaboration of this treatment can be found in Xu and Cai 239 [13]. The final grid resolutions used here are: $d x=0.02 \mathrm{~m}, 0.01 \mathrm{~m}$, and $0.04 \mathrm{~m}$ are used for the 240 near velocity inlet zone, main computational zone, and far field from the main computational 241 zone, respectively; and $d y=0.04 \mathrm{~m}, 0.01 \mathrm{~m}$, and $0.02 \mathrm{~m}$ are used for the air zone, the near water 242 zone, and the deep water zone, respectively. The time step is $0.002 \mathrm{~s}$. The comparison of the 243 wave profiles between the numerical simulations and the analytical results is shown in Fig. 3, 244 where three wave heights, $0.43 \mathrm{~m}, 0.50 \mathrm{~m}$, and $0.63 \mathrm{~m}$, and one SWL, $1.89 \mathrm{~m}$, are considered in 245 order to be consistent with the experimental study by Bradner et al. [9]. Good agreement is 246 obtained as shown in this figure.

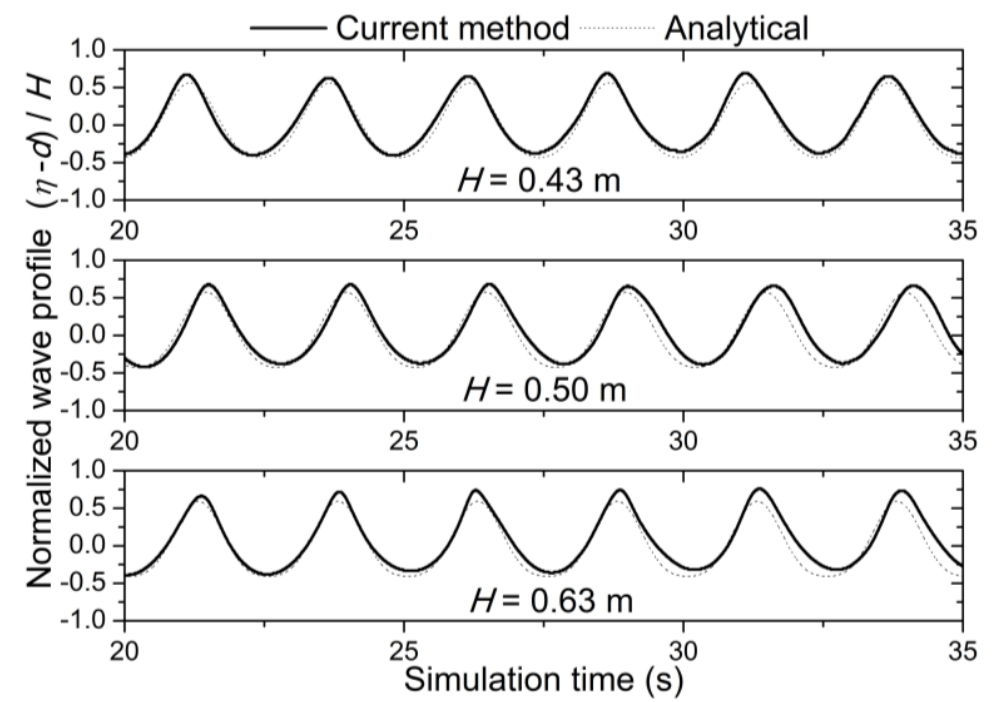

248 Fig. 3 Comparisons of the free surface profiles between the current method and the analytical 249 results 
McPherson [22] considered periodic waves on a 1:20 scaled flat deck, and the result is

252 used here for verification purpose. The experiment was conducted in the Haynes Coastal

253 Engineering Laboratory 3D shallow water wave basin at Texas A\&M University. The flat deck

254 is fixed with $68.58 \mathrm{~cm}$ in width, $106 \mathrm{~cm}$ in length, and $1 \mathrm{~cm}$ in thickness, and the bottom of the

255 flat deck is located $0.48 \mathrm{~m}$ above the bed. During the experimental study, end plates were placed

256 in both ends of the flat deck to eliminate the 3D effects. Here, 3D effects specifically refer to that

257 the wave forces on the two end parts of the bridge deck model may not equal to those on the

258 middle part. Two typical treatments are usually adopted in order to alleviate the 3D effects, i.e.,

259 building a longer model by taking the middle part as the effective part and placing end plates at

260 both ends of the model $[6,22]$.

261 In the verification process, three water depths, $0.48 \mathrm{~m}, 0.51 \mathrm{~m}$, and $0.54 \mathrm{~m}$, are

262 considered with one wave height of $0.14 \mathrm{~m}$, and the computational domain is $13 \mathrm{~m}$ in length $\times$

$2630.9 \mathrm{~m}$ in height. The grid resolutions for this verification are: $d y=0.02 \mathrm{~m}, 0.0025 \mathrm{~m}$ and $0.005 \mathrm{~m}$

264 for the air zone, the near water zone, and the deep water zone, respectively; and $d x=0.005 \mathrm{~m}$,

$2650.0025 \mathrm{~m}$, and $0.02 \mathrm{~m}$ for the near velocity inlet zone, main computational zone, and far field

266 from the main computational zone, respectively.

267 Comparisons between the results by the current method and by McPherson [22] are

268 shown in Fig. 4, where a small difference between the maximum vertical forces is found when

$269 d=0.48 \mathrm{~m}$. One possible reason may be because there are difficulties in keeping the same wave

270 height of $0.14 \mathrm{~m}$ all along the length of the bridge deck model $(1.06 \mathrm{~m})$ in the wave basin.

271 However, good agreements are observed for this verification. 


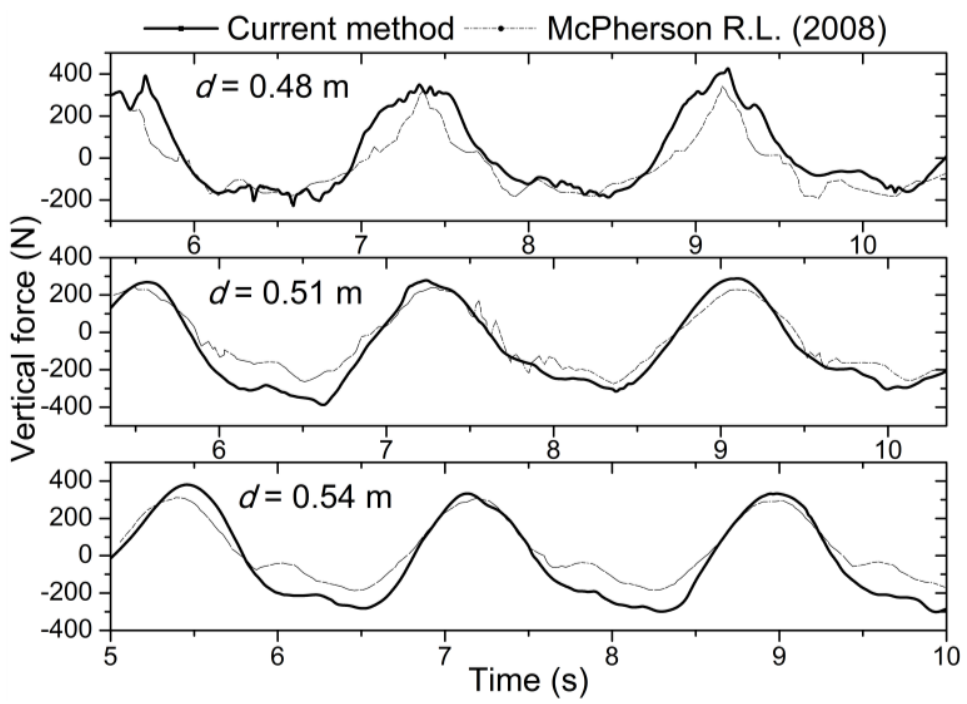

Fig. 4 Comparisons of the time histories of the vertical forces on a flat deck

\section{4}

275

276

277

278

279

\subsection{Deck Force Verification with a Girder Deck Model}

Taking the I-10 Bridge over Escambia Bay as the prototype bridge, a 1:5 scaled bridge deck model is built and tested in the study by Bradner et al. [9]. The geometric parameters for the bridge deck model with some simplifications for the girders are shown in Fig. 5, where the width of the bridge deck is $1.94 \mathrm{~m}$, the girder height is $0.23 \mathrm{~m}$, and the deck depth is $0.05 \mathrm{~m}$. A $0.15 \mathrm{~m}$ (6 inch) steel angle is used in the experimental study to consider the railing effect [23]. The bottom of the bridge deck model is placed at the SWL, i.e., $1.89 \mathrm{~m}$.

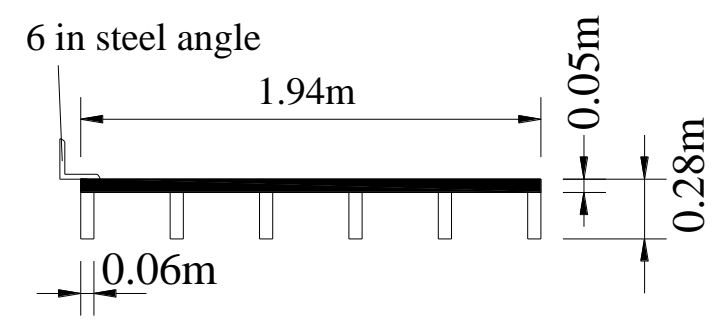

Fig. 5 Dimensions of the simplified bridge deck for verification purpose

In this verification, the 2D numerical domain with the size of $55 \mathrm{~m}$ in length and $3.2 \mathrm{~m}$ in height is used, where the bridge deck model is placed at the middle of the numerical domain. The time histories of the wave forces for two typical wave heights $(0.50 \mathrm{~m}$ and $0.63 \mathrm{~m})$ are shown in 
Fig. 6, where the dashed line refers to the peak values obtained in the experimental

287 measurements by Bradner et al. [9]. The available experimental time history forces are plotted in

288 Fig. 6 (a). It is noted that good agreement for the horizontal forces is obtained, whereas there are

289 some discrepancies for the vertical forces. The reason is probably that the entrapped air in each

290 chamber between two nearby girders underneath the deck plays as a cushion since the air cannot

291 escape in the longitudinal direction in the 2D simulation. This would make the force duration

292 time longer than it should be, i.e., "chubbier" in the pattern than the experimental results. This is

293 one limitation of the 2D simulations. Letters (b) to (f) in Fig. 6 (a) refer to the snapshots of the

294 VOF profiles in the bridge deck-wave interaction, and the corresponding snapshots are shown in

$295 \quad$ Fig. 7.

296

297

298
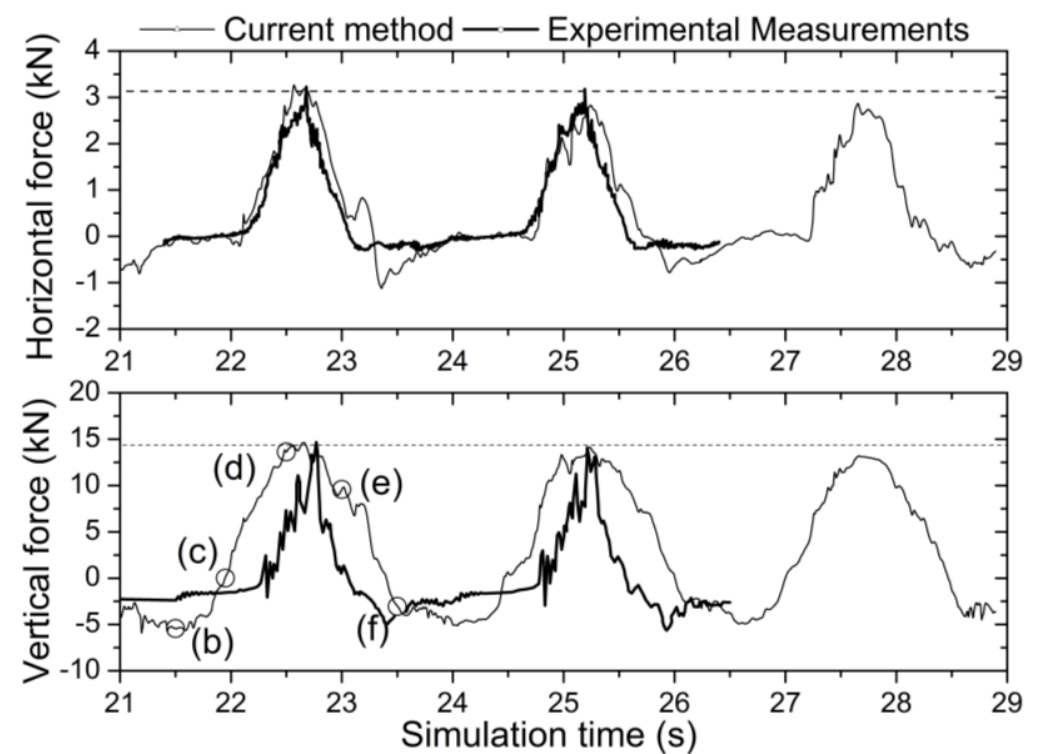

(a) $H=0.50 \mathrm{~m}$ 

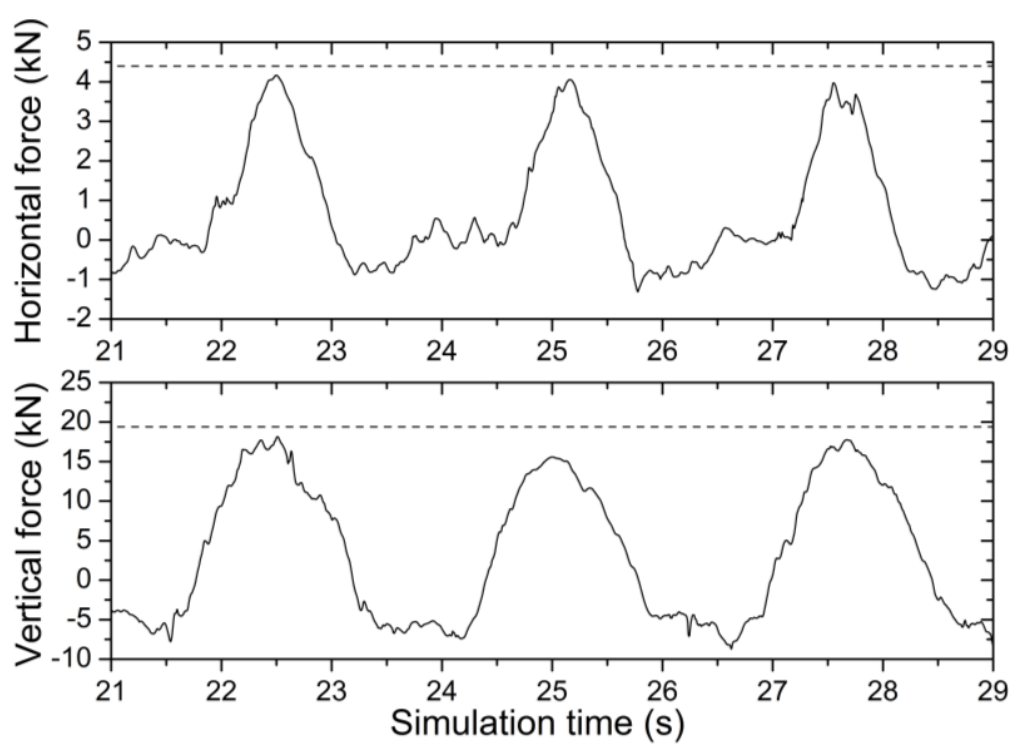

(b) $H=0.63 \mathrm{~m}$

Fig. 6 Comparisons of the time histories of the wave forces on a girder deck. Letters (b) to (f) in Fig. 6 (a) refer to the snapshots of the VOF profiles in the bridge deck-wave interaction, as shown in Fig. 7. The dash line refers to the averaged peak values obtained in the experimental measurements.
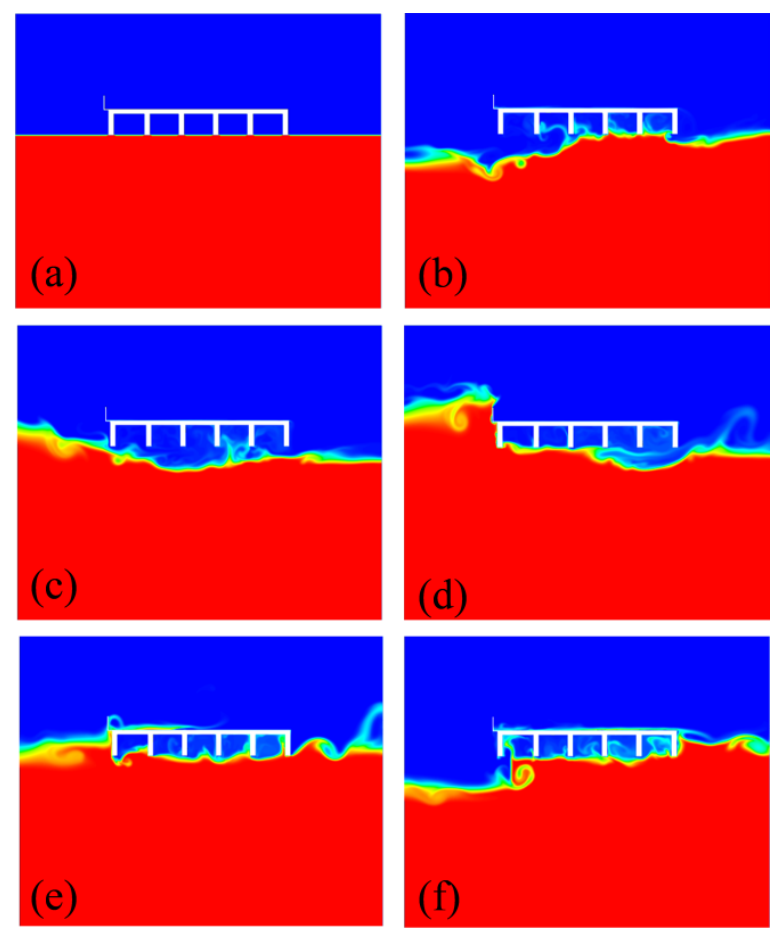

Fig. 7 Snapshots of the VOF profiles in the bridge deck-wave interaction. (a) $t=0.0 \mathrm{~s}$; (b) 
The obtained wave forces by the current method are further compared with the

312 experimental measurements by Bradner et al. [9], and the numerical results by Bozorgnia and

313 Lee [10], as shown in Fig. 8, where five wave heights $(0.20 \mathrm{~m}, 0.34 \mathrm{~m}, 0.43 \mathrm{~m}, 0.50 \mathrm{~m}$, and

$3140.63 \mathrm{~m}$ ) are considered. The wave heights adopted by Bozorgnia and Lee [10] are 0.34 $\mathrm{m}, 0.43 \mathrm{~m}$,

$3150.54 \mathrm{~m}$, and $0.65 \mathrm{~m}$, slightly different with those reported by Bradner et al. [9]. The polynomial

316 relationships between the wave height and the wave forces regressed by Bradner et al. [9], $F_{h}=$

$3172.431 H+6.988 H^{2}$ and $F_{v}=18.01 H+18.69 H^{2}$, are also plotted in Fig. 8, where $F_{h}$ is the horizontal

318 force and $F_{v}$ is the vertical force.

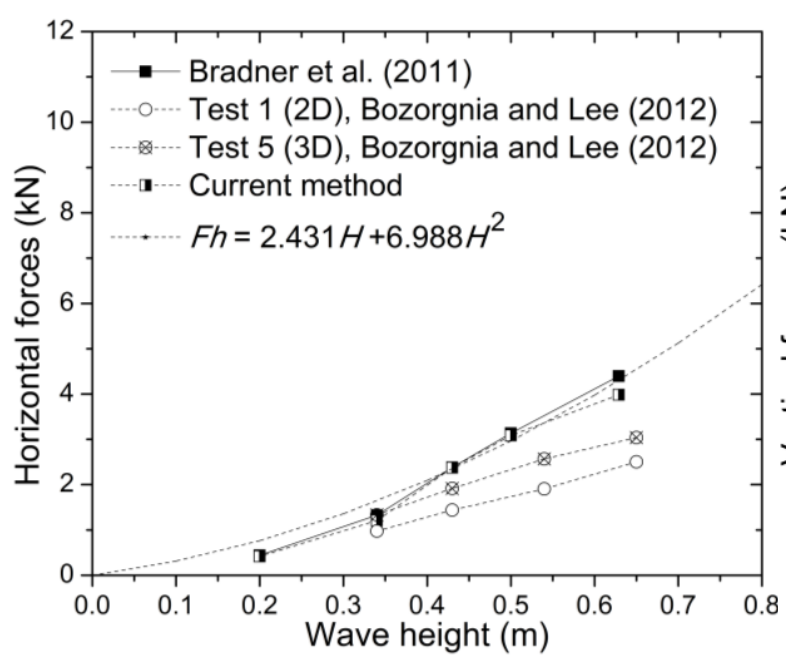

(a) Horizontal force

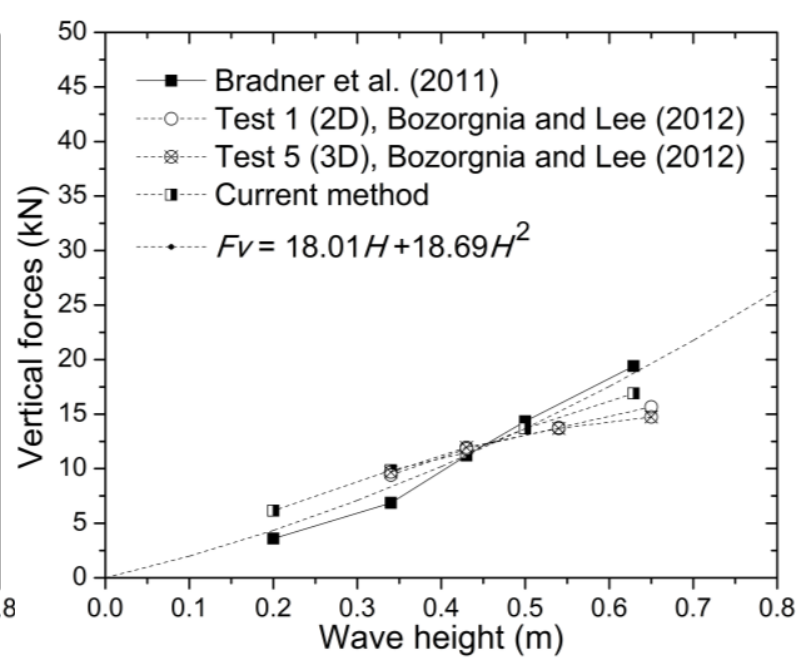

(b) Vertical force

Fig. 8 Comparisons of the wave forces on a girder deck

As noted in Fig. 8, good agreements are found between the results of the current method and those by Bradner et al. [9], especially for the horizontal forces. It is also noted that the 3D simulations (Test 5) by Bozorgnia and Lee [10] estimates larger horizontal wave forces than their corresponding 2D ones (Test 1), whereas close predictions for the vertical wave forces are obtained. However, the horizontal wave forces in both of their $3 \mathrm{D}$ and $2 \mathrm{D}$ simulations are smaller than those by Bradner et al. [9]. The reason Bozorgnia and Lee [10] underestimated the horizontal forces for large wave heights is probably because the $0.15 \mathrm{~m}$ (6 inch) steel angle was 
not considered in their study. For the vertical forces, the current method overestimates the values

331 for small wave heights, which is probably attributed to the entrapped air between the girders

332 underneath the deck slab. In the present 2D simulations, the entrapped air cannot escape in the

333 direction normal to the wave propagating direction (longitudinal direction of the bridge deck),

334 while in the experiment the entrapped air can be released in this direction in a timely manner [10,

$33513,24]$.

\section{3. Parametric Study}

\section{3.1 Determination of the Lateral Restraining Stiffness}

338 In Bradner et al. [9]'s experimental study, to determine the lateral restraining stiffness in

339 the flexible setup, a finite element analysis was conducted to choose the spring stiffness

340 representing the natural frequency ranges in the field condition. Meanwhile, three different

341 elevations of the superstructure, $3.05 \mathrm{~m}, 6.10 \mathrm{~m}$, and $9.14 \mathrm{~m}$ (distance from the mud-line to the

342 bottom soffit of the girders), were chosen. The period of vibrations was then obtained by using 343 the linear-elastic finite element analysis. For example, the fundamental period of one bridge

344 model with the elevation of $9.14 \mathrm{~m}$ and pinned at the foundation, representing the most flexible 345 configuration, was calculated as $0.88 \mathrm{~s}$, corresponding to a model value of $0.40 \mathrm{~s}$ based on the

346 Froude similarity criterion. A suitable support stiffness was thereafter chosen to match this 347 period value in the 1:5 scale model tests.

348 Generally speaking, the lateral restraining stiffness, represented by the elastic springs in

349 Bradner et al. [9]'s study, consists of two parts. One is the substructure stiffness, which depends 350 on the soil condition, the structural stiffness of the piers/piles, etc. The other one is the interface

351 stiffness that depends on the connections between the superstructure and substructure, for 352 example, the bearing types or restraining cables. In the present study, only the total lateral 
353 restraining stiffness of the bridge deck, without distinguishing the substructure stiffness and the

354 interface stiffness, is concerned, i.e., the same as that adopted in Bradner et al. [9]. The Froude

355 similarity criterion is automatically satisfied because full scale bridge models are chosen in the

356 computational domain.

357 For the parametric study, six sets of stiffnesses corresponding to six vibration periods and

358 a damping coefficient $\xi$ of 0.05 are chosen based on the study by Bradner et al. [9], as shown in

359 Table. 1 . In the simulations, the mass is taken as $9716 \mathrm{~kg}$ per unit length according to the study

360 by Xiao et al. [7] (154000 kg/15.85 m=9716 kg per unit length). The case name is defined

361 according to the corresponding value of the stiffness. For example, k1813 refers to the case with

362 the lateral restraining stiffness of $1812.724 \mathrm{kN} / \mathrm{m}$.

Table 1 Parameters for the mass-spring-damper system

\begin{tabular}{cccccc}
\hline Cases & $T_{s}(\mathrm{~s})$ & $m(\mathrm{~kg})$ & $\xi$ & $k_{S}(\mathrm{~N} / \mathrm{m})$ & $c\left(\mathrm{~N} \cdot \mathrm{s}^{2} / \mathrm{m}\right)$ \\
\hline $\mathrm{k} 1813$ & 0.46 & 9716 & 0.05 & 1812724 & 13271 \\
$\mathrm{k} 998$ & 0.62 & 9716 & 0.05 & 997847 & 9846 \\
$\mathrm{k} 630$ & 0.78 & 9716 & 0.05 & 630461 & 7827 \\
k425 & 0.95 & 9716 & 0.05 & 425011 & 6426 \\
k170 & 1.5 & 9716 & 0.05 & 170477 & 4070 \\
k96 & 2.0 & 9716 & 0.05 & 95893 & 3052 \\
\hline
\end{tabular}

The wave height considered is $2.0 \mathrm{~m}$, the water depth is $8.4 \mathrm{~m}$, and the wave period is 5.5

$366 \mathrm{~s}$ [25]. The computational domain is $10 \mathrm{~m}$ in height and $200 \mathrm{~m}$ in length. The geometry of the

367 bridge deck model is shown in Fig. 2 (a) earlier. Six different structure elevations are considered

368 to simulate in field conditions, as listed in Table 2, where $C_{S}\left(C_{S}=S / H_{b}\right)$ is the coefficient of

369 submersion depth and is negative when the bottom of the superstructure is under the SWL; $S=$

370 the distance from the bottom of the bridge superstructure to the SWL; and $H_{b}=$ superstructure

371 depth. The momentum center is the moment center due to the vertical force and the horizontal

372 force, and it is located at the middle height of the deck for each case. The abbreviation name of

373 each case is designated according to both the bridge elevation (the value refers to the elevation of 
374 the bottom of the girder) and the submersion coefficient; for example, E8.4/CS(0) stands for the

375 case when the bottom of the bridge model is $8.4 \mathrm{~m}$ from the sea bed and the corresponding

376 coefficient of submersion depth $C_{S}$ is 0.

Table 2 Structure elevations and corresponding coefficients

\begin{tabular}{cccccc}
\hline Cases & $\begin{array}{c}\text { Bridge elevation } \\
\text { (Bottom of the } \\
\text { girder) }(\mathrm{m})\end{array}$ & $S(\mathrm{~m})$ & $C_{s}=S / H_{b}$ & $\mathrm{x}(\mathrm{m})$ & $\mathrm{y}(\mathrm{m})$ \\
\hline E9.0/CS(0.444) & 9.0 & 0.6 & 0.444 & 95.225 & 10.2 \\
E8.7/CS(0.222) & 8.7 & 0.3 & 0.222 & 95.225 & 9.9 \\
E8.4/CS(0) & 8.4 & 0 & 0 & 95.225 & 9.6 \\
E8.1/CS(-0.222) & 8.1 & -0.3 & -0.222 & 95.225 & 9.3 \\
E7.8/CS(-0.444) & 7.8 & -0.6 & -0.444 & 95.225 & 9.0 \\
E7.5/CS(-0.667) & 7.5 & -0.9 & -0.667 & 95.225 & 8.7 \\
\hline
\end{tabular}

378

\subsection{Structural Vibration}

380 One example of the bridge deck displacement is shown in Fig. 9 where the case of 381 E7.8/CS(-0.444) with different restraining stiffnesses starts from the original position of the 382 bridge deck, $95.225 \mathrm{~m}$, when the simulation begins. The vibration amplitude of Case $\mathrm{k} 96$ is 383 relatively larger than those of other cases with higher stiffnesses. The maximum bridge deck 384 displacements for all the six structure elevations with different restraining stiffnesses are shown 385 in Fig. 10. The displacement values (in the landward direction) show the same trends, namely, 386 the less restraining stiffness, the larger the deck displacement. For a given stiffness of all the six 387 cases, the deck displacement differs from each other, which is also related to the values of the 388 horizontal forces. This is the same as observed for solitary wave actions on flexible bridge decks 389 [13]. 


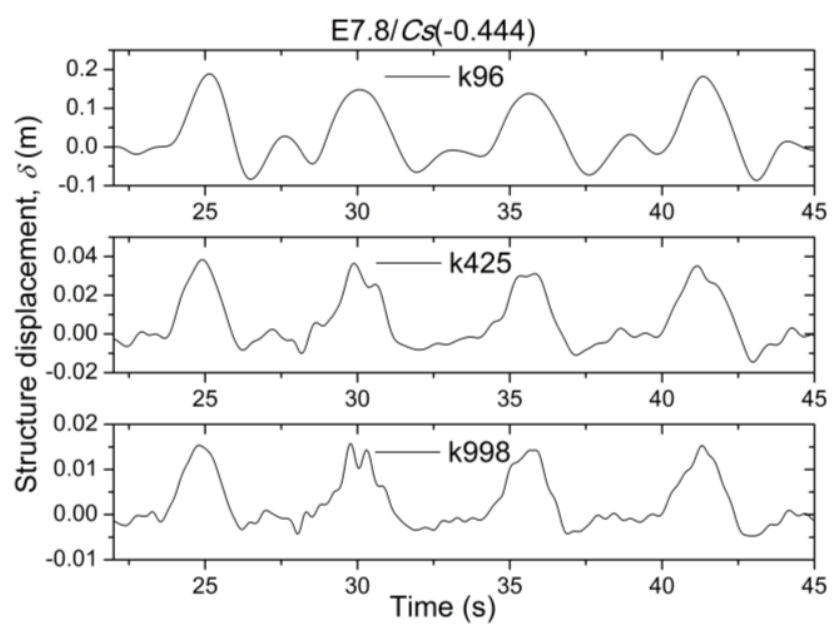

Fig. 9 Structure displacement for Case E7.8/CS(-0.444)

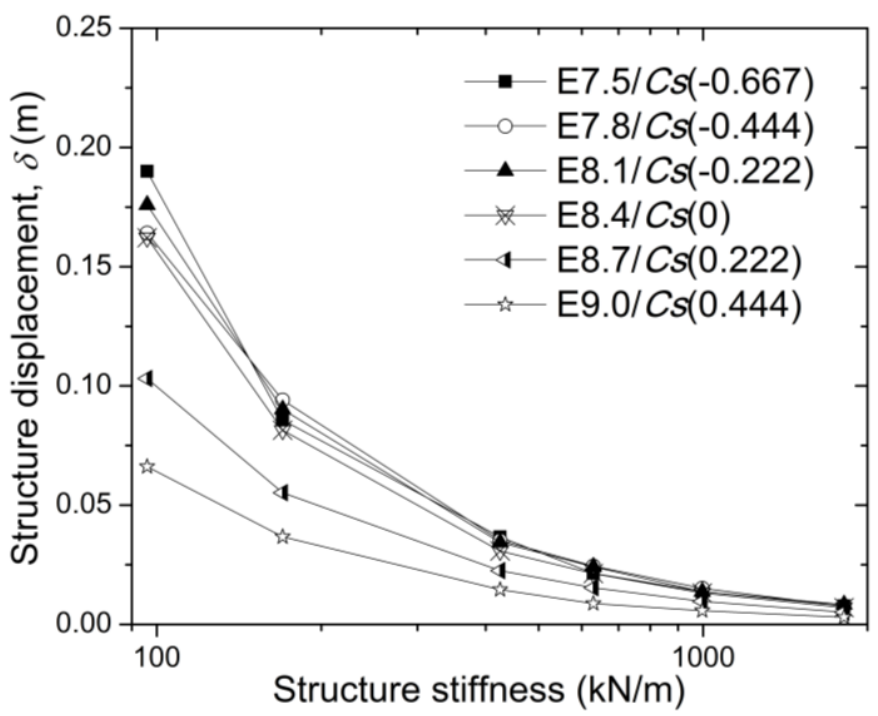

Fig. 10 Maximum displacement of the structural vibration

\subsection{Horizontal Forces without considering Inertia Forces}

Wave forces on coastal structures generally refer to the net hydraulic forces without

396 including the inertia forces from the bridge deck, i.e., the horizontal force $F(t)$ in Eq. (1). These

397 kinds of wave forces are usually used to design and evaluate the performance of the bridge 398 superstructure. One example of the time-history horizontal forces without considering inertia 399 forces in Case E7.8/CS(-0.444) is shown in Fig. 11. It can be observed in this figure that the 400 time-history force curves slightly differ from each other with different structure stiffnessnes. The 
401 positive horizontal forces, $F_{h}$, obtained from the time histories versus the restraining stiffness are

402 plotted in Fig. 12 (a), and a normalized expression, $\frac{F_{h}}{F_{h k 1813}}$, is used to demonstrate the general 403 trends of the horizontal forces, as shown in Fig. 12 (b). Here $F_{h k 1813}$ is the value of the positive 404 horizontal force corresponding to the restraining stiffness of $1812.724 \mathrm{kN} / \mathrm{m}$. It can be seen in 405 Fig. 12 (b) that the horizontal forces vary along with the increase of the structure stiffness, but 406 without a clear general trend. This is slightly different from the case of solitary wave actions on 407 the flexible bridge decks reported by $\mathrm{Xu}$ and Cai [13], where the values using this normalization 408 generally decrease and approach 1 with the increase of the restraining stiffness.

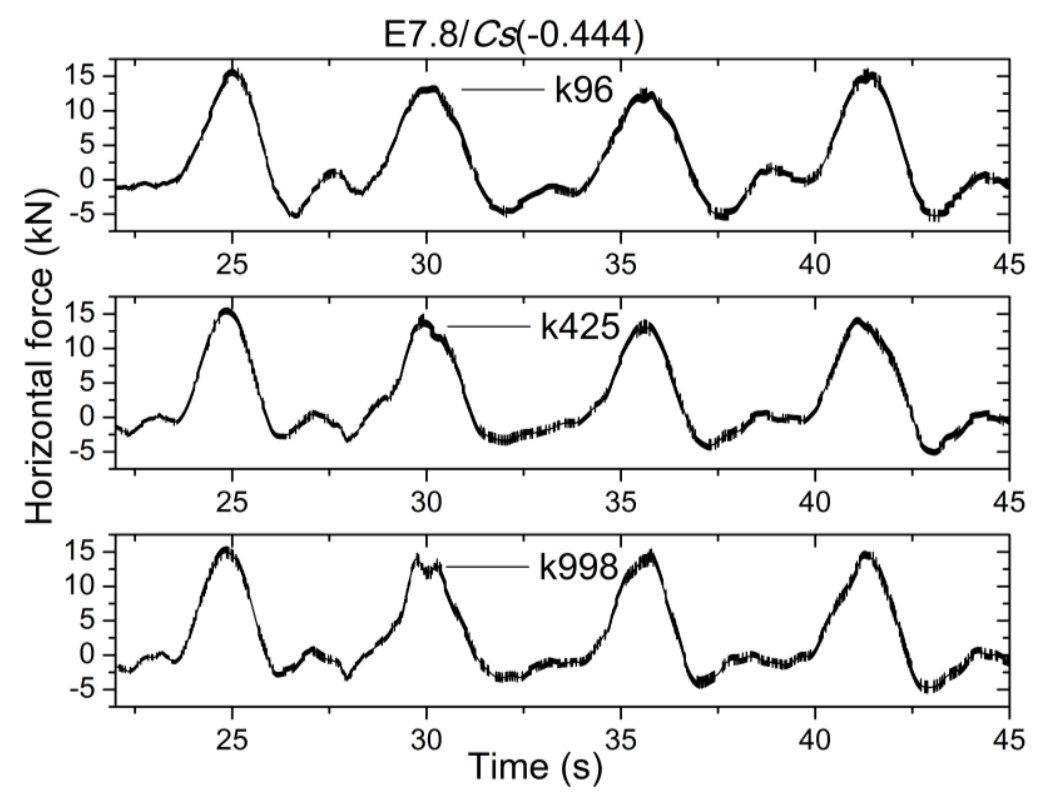

Fig. 11 Time histories of the horizontal forces for Case E7.8/CS(-0.444) 


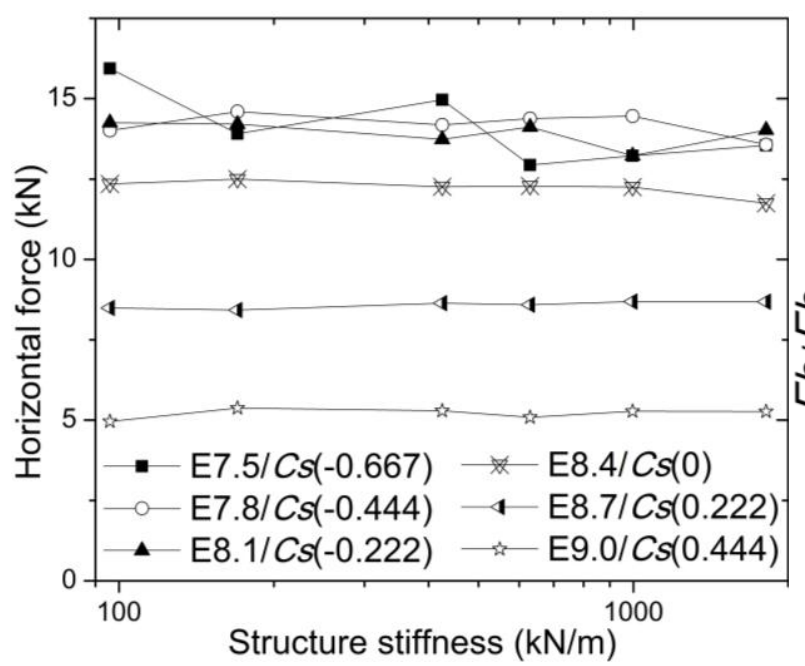

(a) Before normalization

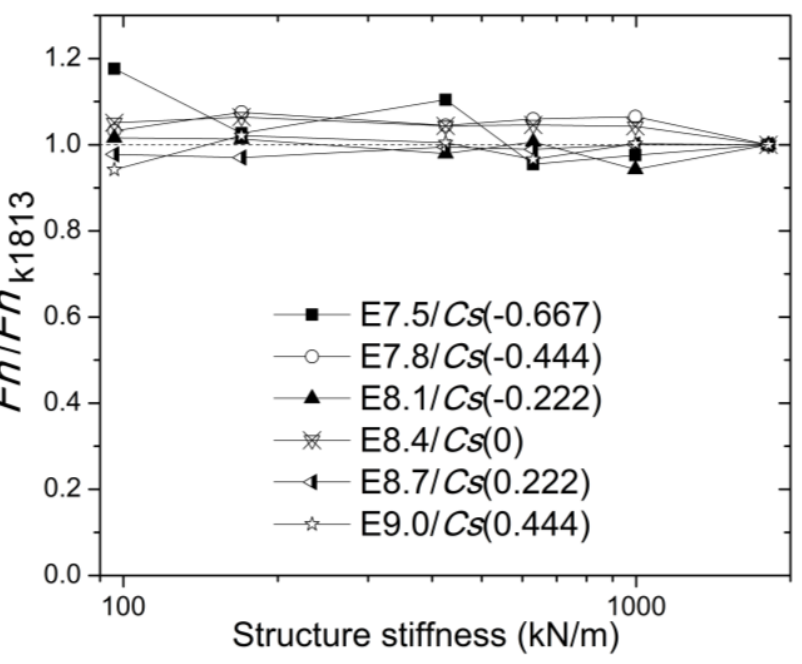

(b) After normalization
413

414

415

416

417

418

419

420

421

422

423

424

425

426

427

428

429

430

Fig. 12 Horizontal forces considering different restraining stiffnesses

\subsection{Horizontal Forces with considering Inertia Forces}

The horizontal forces with considering inertia forces from the bridge deck are those transferred from the bridge deck to the interface and substructure. Hence, they should be used to design and evaluate the bearing supporting and the substructure. The difference between the horizontal force with and without considering the inertia forces can be expressed as the difference between $\boldsymbol{F}(\boldsymbol{t})$ and $\boldsymbol{F}(\boldsymbol{t})-\boldsymbol{m} \ddot{\boldsymbol{x}}$, where $-\boldsymbol{m} \ddot{\boldsymbol{x}}$ is the inertia forces of the bridge superstructure, see Eq. (1). For fixed bridge decks, there is no difference between $\boldsymbol{F}(\boldsymbol{t})$ and $\boldsymbol{F}(\boldsymbol{t})$ -

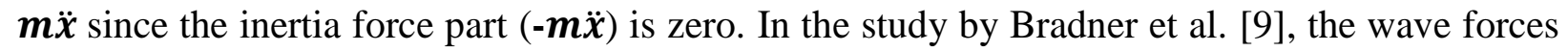
were collected from the load cells and hence, the horizontal forces they obtained for the flexible setups are actually the forces including the inertia forces. In the following discussions, the inertia force part is expressed as $\boldsymbol{F}_{\boldsymbol{I}}$.

Fig. 13 shows an example of the comparisons of the time-history horizontal forces with and without considering inertia forces for Case E8.1/CS(-0.222). From this figure, obvious differences can be found when the restraining stiffness is relatively small (k96). However, for large restraining stiffness (k1813), the curves for the two types of horizontal forces almost 
431 coincide with each other, indicating that the inertia force in this specific case is relatively small.

432 This observation is attributed to that more flexible setups tend to have larger accelerations for the

433 bridge deck under the horizontal wave forces in the same magnitude and hence result in larger

434 inertia forces for the bridge deck. The inertia forces are then transferred from the bridge deck to 435 the interface and substructure.

436

437

440

441

442

443 considering inertia forces. As shown in Fig. 14 (a), the proportion caused by the inertia force

444 (related to acceleration) can be clearly identified as discussed above, especially for more flexible

445 setups. However, other factors, while not well determined, also add credits for this proportion.

446 Specifically speaking, the horizontal forces without considering inertia forces are not constant

Fig. 13 Example of the comparisons of the time-history horizontal forces with and without considering inertia forces for Case E8.1/CS(-0.222)

A typical comparison of the horizontal forces with and without considering the inertia forces for different stiffnesses is shown in Fig. 14. It shows that with the increase of the restraining stiffness, these two types of horizontal forces tend to be closer with smaller difference. In addition, the horizontal forces with considering inertia forces are larger than those without

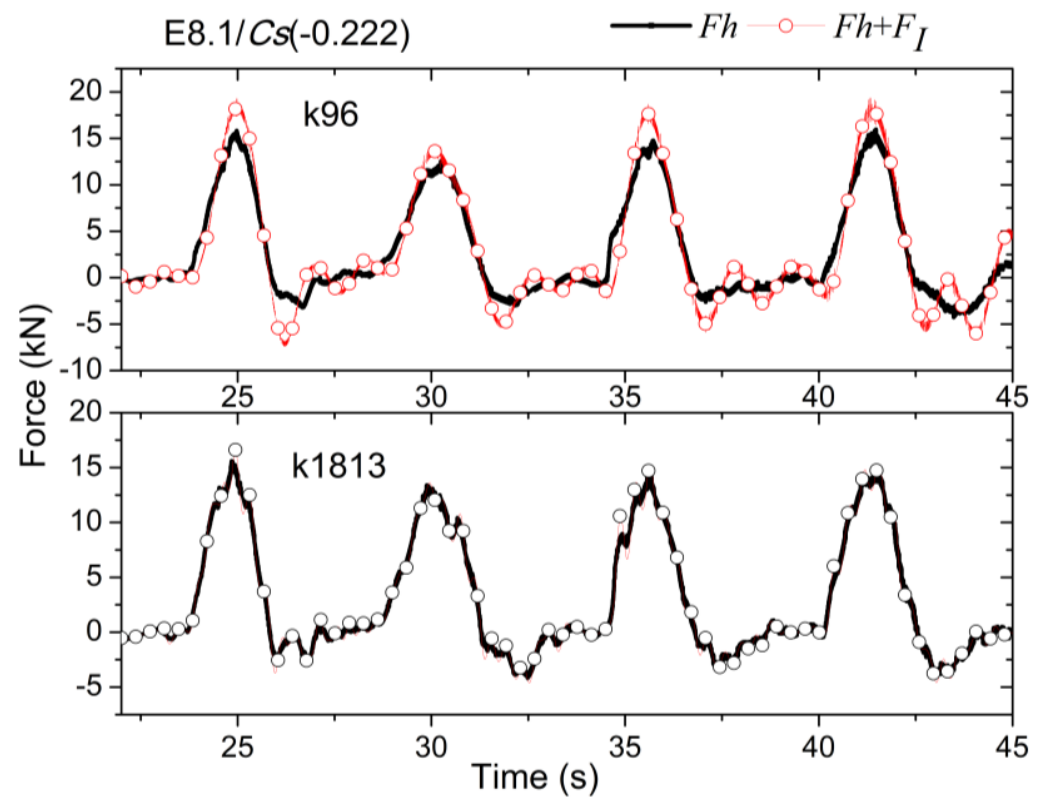


447 for different structure stiffness. Their variance along with different restraining stiffnesses is 448 probably due to that: (1) The positive horizontal forces obtained in the current study are averaged

449 over four wave periods. Long time simulations would produce more stable results per the 450 averaging procedure and hence, less variance would be predicted for the different restraining 451 stiffnesses; and (2) The bridge deck vibration may have certain effects on the horizontal forces.

452 In Fig. 14 (b), the value of $\frac{F_{h}+F_{I}}{F_{h}}$ is 1.2 for Case $\mathrm{k} 96$, indicating that when the bridge 453 superstructure is allowed to vibrate with a period of $2.0 \mathrm{~s}$, an increment of 20 percent of the 454 horizontal wave forces will be transferred to the bearing (interface) and the substructure. Similar 455 observations were obtained in $\mathrm{Xu}$ and Cai [13] for solitary waves, but the quantitative results are 456 quite different.

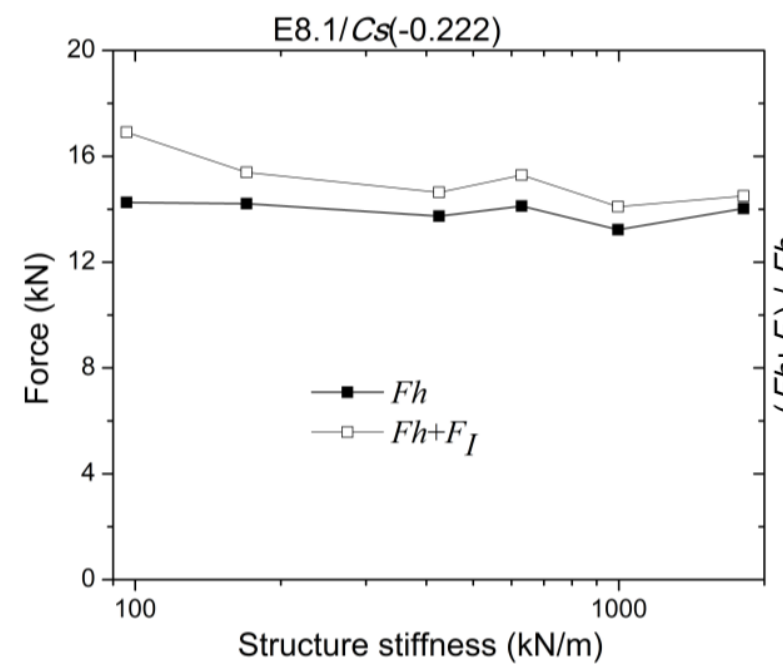

(a) Before normalization

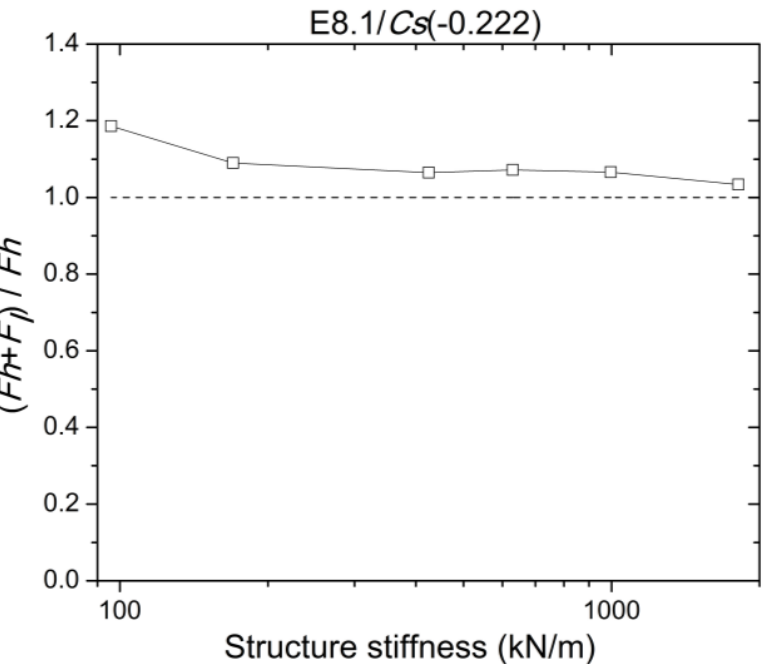

(b) After normalization

Fig. 14 One example of the comparisons of the horizontal forces with and without considering inertia forces

\subsection{Vertical forces}

One example of the time-history vertical forces for Case E7.8/CS(-0.444) is shown in Fig.

15. The obtained vertical forces versus the restraining stiffness are plotted in Fig. 16(a) and, similarly, the vertical forces are normalized using an expression of $\frac{F_{v}}{F_{v k 1813}}$, as shown in Fig. 
16(b), where $F_{v k 1813}$ is the vertical force corresponding to the restraining stiffness of 1812.724

$467 \mathrm{kN} / \mathrm{m}$. The negative vertical forces are not discussed here since they are significantly smaller

468 than the positive vertical forces. It is observed in Fig. 16 that the vertical forces do not have a

469 clear general trend with the increase of the restraining stiffness. The values of $\frac{F_{v}}{F_{v k 1813}}$ are quite

470 close to 1 for the studied cases, showing that increasing the structural flexibilities does not have

471 obvious benefits to reducing the vertical forces during the bridge deck-wave interaction. This

472 confirms the observation in $\mathrm{Xu}$ and Cai [13].

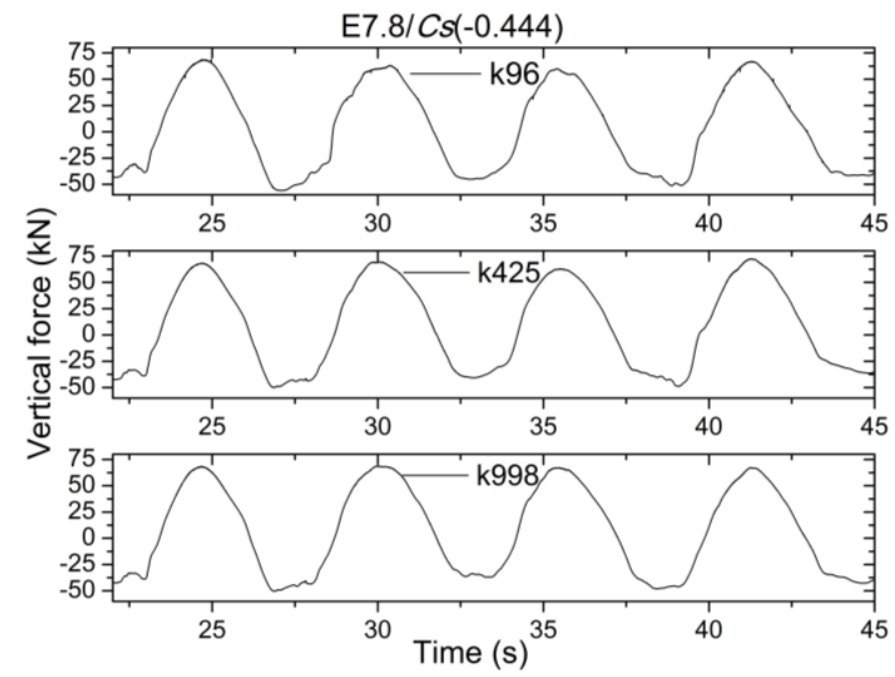

Fig. 15 Time histories of the horizontal forces for Case E7.8/CS(-0.444)
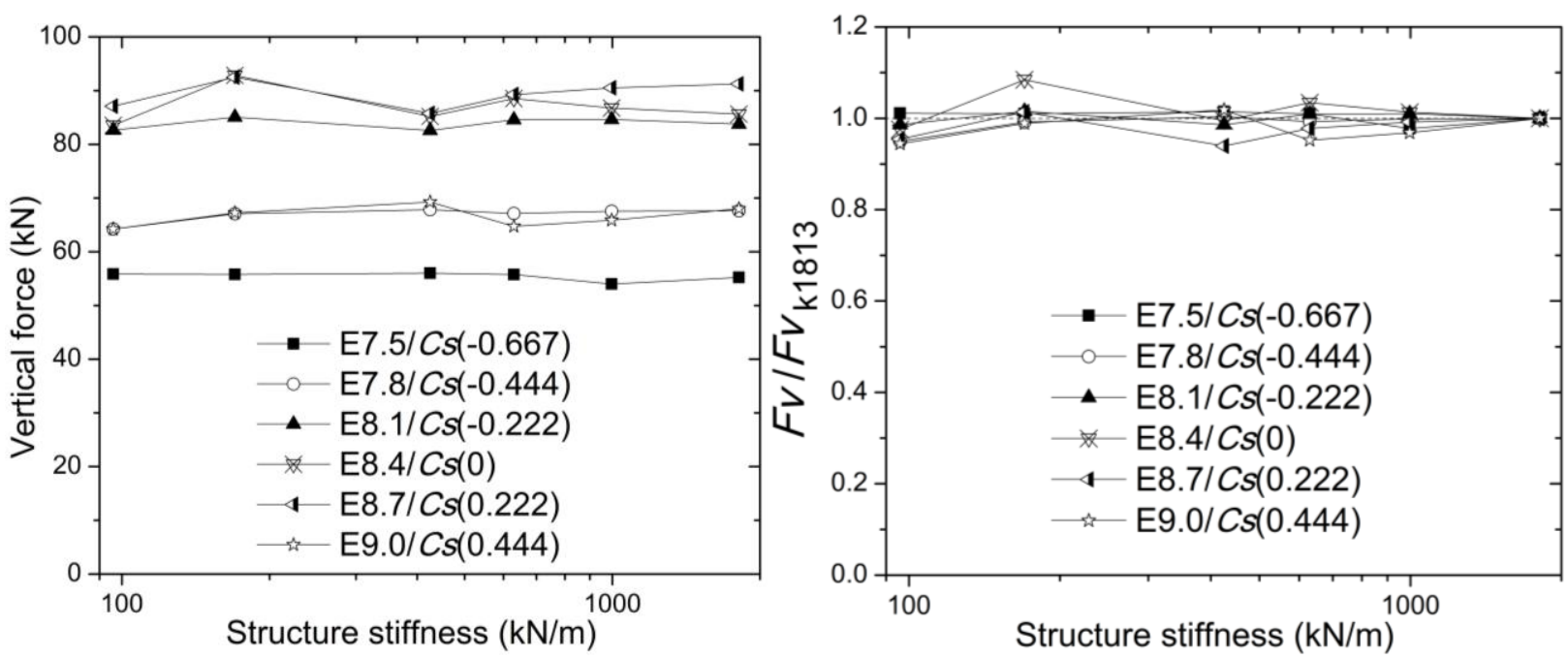
$486 \quad c_{a m p}=\frac{k * \delta}{F_{h}}$

488 figure that the values of $c_{a m p}=\frac{k * \delta}{F_{h}}$ are larger than 1.0 and the general trends tend to be closer to

4891.0 as the restraining stiffness increases. It is predicted that as the restraining stiffness goes to

490 infinity, the value of $c_{a m p}=\frac{k * \delta}{F_{h}}$ should be closer to 1.0. The same trend is reported in $\mathrm{Xu}$ and

491 Cai [13], but the values of $c_{a m p}=\frac{k * \delta}{F_{h}}$ are quite different between the results by these two types 492 of waves.
(a) Before normalization
(b) After normalization

Fig. 16 Vertical forces considering different restraining stiffnesses

\subsection{Dynamic Amplification Factor}

It is well known that in the static structural analysis, $\delta=\frac{F}{k}$, where $\delta$ is the structure displacement, $F$ is the force applied in the direction of the structure displacement, and $k$ is the structure stiffness. For the bridge deck-wave interaction under Stokes $2^{\text {nd }}$ order waves, the dynamic effect (the amplification factor) needs to be considered, where the amplification factor is expressed as follows:

$$
c_{a m p}=\frac{k * \delta}{F_{h}}
$$

The results of the amplification factor are plotted in Fig. 17. It can be observed in this 
Fig. 17 The dynamic amplification factor, $c_{a m p}$, versus the restraining stiffness

\subsection{Discussion of the Setups in the Laboratory Studies regarding Lateral Stiffness}

In the laboratory studies for predicting the wave forces on bridge deck models, an

497 important problem usually exists that there is certain lateral flexibility for the supporting

498 structures [6, 26]. One typical experimental setup in the literature is shown in Fig. 18, where the

499 support rods cannot be deemed as rigid structures and they will probably vibrate under the wave

500 impacts. As such, the wave loads obtained from the load cell should include the proportion of the

501 inertia forces, and the vibration of the bridge deck may have some effects on the values of the as-

502 obtained wave forces. Sheppard and Marin [6] proposed a more rigid setup for developing the

503 AASHTO code [5] and the load cell was placed very adjacent to the bridge deck model. Though

504 the supporting steel frame would vibrate at a certain frequency, the load cell should vibrate along

505 with the bridge deck model. Nevertheless, the support structure for acquiring the data in

506 developing the AASHTO code [5] can be considered as a rigid setup. Therefore, attentions

507 should be paid to the differences of the horizontal forces with and without considering the inertia

508 forces since the deck vibrations cannot be avoided under infield conditions.
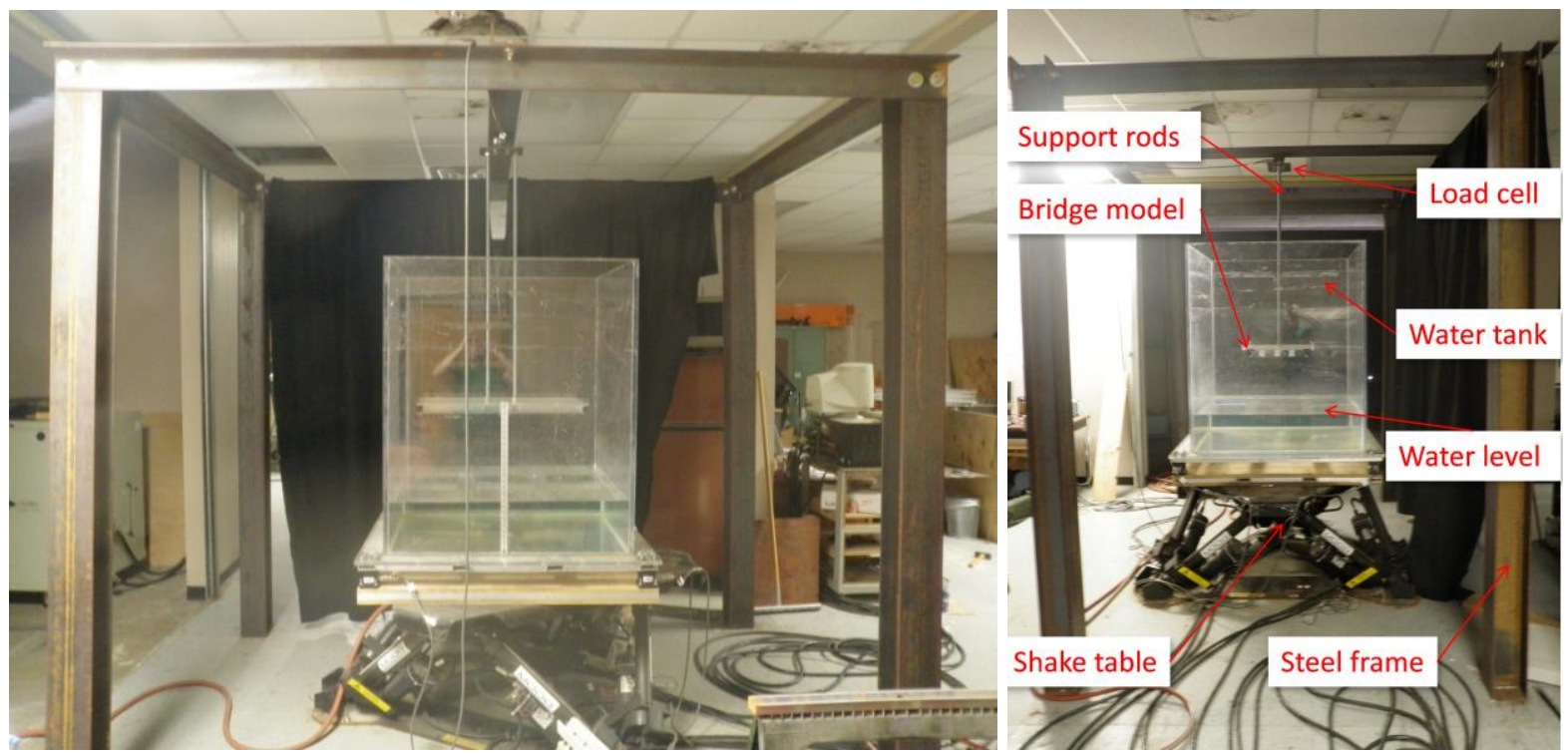


\section{4. Concluding Remarks}

513 This paper has addressed one fundamental question on the bridge deck-wave interaction:

514 do the bridge deck vibrations result in smaller wave forces on deck? Based on a methodology of

515 the mass-spring-damper system using a dynamic mesh updating technique proposed in the

516 previous studies, a wave model of the Stokes $2^{\text {nd }}$ order wave theory is applied and verified first

517 with analytical solutions and experimental measurements in the literature. The SST $k$ - $\omega$ model is

518 used as the turbulence closure for the RANS equations in order to capture the turbulence effects

519 in the bridge deck-wave interaction. A parametric study was then conducted to investigate the

520 characteristics of the bridge deck-wave interaction for different structure elevations with

521 different lateral restraining stiffnesses. Based on the numerical predictions, the following

522 conclusions can be drawn.

523 (1) For the bridge superstructures with different elevations studied in the present study, a 524 smaller restraining stiffness tends to result in a larger deck displacement as expected.

525 (2) When the inertia forces are ignored, there is not much difference for the horizontal 526 forces and vertical forces with the variable restraining stiffness. Therefore, increasing the 527 structural flexibilities in the lateral/transverse direction does not necessarily benefit the bridge 528 structure with an obvious force reduction for both the horizontal and vertical forces on the bridge 529 superstructure.

530 (3) With the increase of the structural flexibilities, larger differences can be found 531 between the horizontal forces with and without considering the inertia forces. It is noted that in 532 the development of the AASHTO [5] guide specifications [6], the support structure for acquiring 533 the data was considered as a rigid setup. As such, attentions should be paid to the differences of 
534 the horizontal forces with and without considering the inertia forces since the deck vibrations 535 cannot be avoided under infield conditions.

536 (4) The amplification factor $c_{a m p}$ is larger than 1.0 as predicted for the horizontal forces 537 with different structure elevations. In addition, the amplification factor tends to be closer to 1.0 538 as the restraining stiffness increases.

539 (5) In general, compared with the observations for solitary wave actions on flexible 540 bridge decks [13], a slight difference exists for the general trends of the horizontal forces versus 541 the restraining stiffness. Other than this, these two kinds of wave types share almost the same 542 general trends for the considered aspects, but different result values.

543 The limitations of the current study and future work are described as follows:

544 (1) The methodology for the 2D numerical simulations employed in the current study 545 may not fully capture all the features in the bridge deck-wave interaction process. The entrapped 546 air in each chamber between two nearby girders underneath the deck, playing as a cushion 547 between the water surface and the bottom of the bridge deck, is an issue in the $2 \mathrm{D}$ simulations 548 since the entrapped air cannot escape in the longitudinal direction. Therefore, improvements and 549 more realistic results would be expected in the 3D simulations for both the fixed and flexible 550 setups. However, valid 3D simulations with sufficient fine model meshes require much more 551 powerful computational capabilities.

552 (2) A countermeasure of air venting holes in the bridge deck-wave interaction is 553 identified to in favor of reducing the vertical force substantially, but an increase of the horizontal 554 force is therefore resulted [15]. The bridge deck-wave interaction with considering both the 555 flexible setups and air venting holes would be a topic of interest and need to be further 556 systematically studied. 


\section{Acknowledgement}

This work was supported by Louisiana State University, under the Economic

Development Assistantship for the first author and the NSF Grant CMMI-0927824 for the second author. The work is also partially supported by HPC@LSU high performance computing resources. All of the opinions presented here are those of the writers, not necessarily representing

562 those of the sponsors.

References

[1] Graumann A, Houston T, Lawrimore J, Levinson D, Lott N, McCown S, Stephens S, Wuerts D. Hurricane Katrina: A climatological perspective-Preliminary report. Technical Rep. No. 2005-01, NOAA's Climate Data Center, Washington D.C; 2005.

[2] Robertson IN, Riggs HR, Yim SCS, Young YL. Lessons from Hurricane Katrina Storm Surge on Bridges and Buildings. J of Waterway, Port, Coastal, and Ocean Engineering, ASCE 2007; 133(6), 463-483.

[3] Okeil AM, Cai CS. Survey of short- and medium-span bridge damage induced by Hurricane Katrina. J of Bridge Engineering 2008; 13(4), 377-387.

[4] Padgett JE, DesRoches R, Nielson BG, Yashinsky M, Kwon OS, Burdette N, Tavera E. Bridge Damage and Repairs Costs from Hurricane Katrina. J of Bridge Engineering 2008; 13(1), 6-14.

[5] AASHTO. Guide Specifications for Bridges Vulnerable to Coastal Storms. American Association of State Highway and Transportation Officials, Washington D.C; 2008.

[6] Sheppard DM, Marin J. Wave loading on bridge decks. Final Report Submitted to Florida Department of Transportation, Gainesville, FL; 2009.

[7] Xiao H, Huang W, Chen Q. Effects of submersion depth on wave uplift force acting on Biloxi Bay Bridge decks during Hurricane Katrina. Computer \& Fluids 2010; 39, 13901400.

[8] Jin J, Meng B. Computation of wave loads on the superstructure of coastal highway bridges. Ocean Engineering 2011; 38, 2185-2200.

[9] Bradner C, Schumacher T, Cox D, Higgins C. Experimental Setup for a Large-Scale Bridge Superstrcuture Model Subjected to Waves. Journal of Waterway, Port, Coastal, and Ocean Engineering, ASCE 2011; Vol. 137. No. 1, 3-11.

[10] Bozorgnia M, Lee JJ. Computational Fluid Dynamic Analysis of Highway Bridges exposed to Hurricane Waves. Proceedings of $33^{\text {rd }}$ Conference on Coastal Engineering, Santander, Spain; 2012.

[11] Guo A, Fang Q, Bai X, Li H, Hydrodynamic experiment of the wave force acting on the superstructures of coastal bridges. Journal of Bridge Engineering 2015; 20(12), 04015012.

[12] Guo A, Fang Q, Li H. Analytical solution of hurricane wave forces acting on submerged bridge decks. Ocean Engineering 2015; Vol. 108, 519-528. 
[13] Xu G, Cai CS. Numerical Simulations of Lateral Restraining Stiffness Effect on Bridge Deck -Wave Interaction under Solitary Waves. Engineering Structures 2015; 101, 337351.

[14] Xu G, Cai CS, Han Y. Investigating the Characteristics of the Solitary Wave Induced Forces on Coastal Twin Bridge Decks. Journal of Performance of Constructed Facilities 2015; 10.1061/(ASCE)CF.1943-5509.0000821, 04015076.

[15] Xu G, Cai CS, Chen Q. Countermeasure of Air Venting Holes in the Bridge Deck -Wave Interaction under Solitary Waves. Journal of Performance of Constructed Facilities 2016; 10.1061/(ASCE)CF.1943-5509.0000937, 04016071.

[16] Hayatdavoodi M, Ertekin .C. Nonlinear wave loads on a submerged deck by the GreenNaghdi equations. Journal of Offshore Mechanics and Arctic Engineering 2015; 137(1), 011102. doi: 10.1115/1.4028997.

[17] ANSYS ${ }^{\circledR}$ Academic Research, Release 15.0.

[18] Huang W, Xiao H. Numerical Modeling of Dynamic Wave Force Acting on Escambia Bay Bridge Deck during Hurricane Ivan. Journal of Waterway, Port, Coastal, and Ocean Engineering, ASCE 2009; 135(4), 164-175.

[19] Sarpkaya T, Isaacson M. Mechanics of wave forces on offshore structures. Van Nostrand Reinhold, New York; 1981.

[20] Hirt CW, Nichols BD. Volume of fluid (VOF) method for the dynamics of free boundaries. Journal of Computational Physics 1981; 39(1), 201-225.

[21] Guo X, Wang B, Liu H, Miao G. Numerical Simulation of Two-Dimensional Regular Wave Overtopping Flows over the Crest of a Trapezoidal Smooth Impermeable Sea Dike. J. Waterway, Port, Coastal, Ocean Eng. 2014; 10.1061/(ASCE)WW.1943-5460.0000234, 04014006.

[22] McPherson RL. Hurricane Induced Wave and Surge Forces on Bridge Decks. M.S. thesis, Texas A\&M University, College Station, TX; 2008.

[23] Bradner C. Large-Scale laboratory observations of wave forces on a highway bridge superstructure. M.S. thesis, Dept. of Civil and Construction Engineering, Oregon State Univ., Corvallis, Ore; 2008.

[24] Xu G, Cai CS. Wave forces on Biloxi Bay Bridge Decks with inclinations under solitary waves. Journal of Performance of Constructed Facilities 2015; 29(6), 04014150.

[25] Chen Q, Wang L, Zhao H. Hydrodynamic investigation of coastal bridge collapse during Hurricane Katrina. J. Hydraul. Eng. 2009; 135(3),175-186.

[26] Henry AM. Wave Forces on Bridge Decks and Damping Techniques to Reduce Damages. M.S. thesis, Louisiana State University, Baton Rouge, LA; 2011. 